\title{
LA MODERNIZACIÓN ADMINISTRATIVA DEL GOBIERNO DE FELIPE CALDERÓN: ENTRE LA AUSTERIDAD Y LA REFORMA
}

María del Carmen Pardo

\section{INTRODUCGIÓN}

LOS PROCESOS DE MODERNIZACIÓN ADMINISTRATIVA han sido impulsados de manera distinta por cada gobierno según estrategias y programas con sellos distintivos.* A partir del gobierno de Luis Echeverría se empiezan a observar reformas importantes al aparato administrativo del gobierno federal; sin embargo, no es hasta las elecciones federales de 2000, con el cambio de partido en el ejecutivo federal, que el proceso de modernización administrativa se inscribe dentro de un sistema político más plural, en el que por primera vez después de setenta años las decisiones en el ejecutivo federal no emanaban de una actuación hegemónica.

De ahí que la alternancia política en 2000 también levantara mayores expectativas en torno a una profunda reforma modernizadora del gobierno la cual debía conducir a una también profunda transformación de la relación de éste con los ciudadanos. Durante la administración de Vicente Fox la agenda de modernización administrativa fue ambiciosa y se sustentó en una apuesta por adoptar en el sector público algunas de las prácticas del sector privado, haciendo énfasis en la Nueva Gerencia Pública (NGP), en la transformación de la relación del gobierno con los ciudadanos al intentar satisfacer mejor sus demandas y en la promoción de mecanismos artículo.

* Agradezco el apoyo de Miguel Ángel Berber para la elaboración de este 
para fomentar su participación directa. Sin embargo, la envergadura de este proyecto requería un liderazgo político e institucional claro, sostenido y con un bien definido esquema de implementación (en términos del tiempo y de los actores necesarios para su puesta en marcha), condiciones que no estuvieron presentes a lo largo de ese gobierno, si bien se impuso de manera acrítica el énfasis por adoptar prácticas del sector privado en las instituciones gubernamentales.

El gobierno de Felipe Calderón no tuvo como prioridad impulsar estos procesos modernizadores, aunque sí se tomaron algunas decisiones para mejor el desempeño de la administración pública federal, siempre mediadas por otras acciones. La importancia del proyecto de modernización administrativa fue mucho menos evidente en este gobierno; se volvió a insistir en la necesidad de perfeccionar instrumentos como el presupuesto basado en resultados, la evaluación del desempeño y la planeación estratégica a partir de metodologías estandarizadas, como la Matriz de Marco Lógico. No obstante, la atención gubernamental hacia los problemas en los ámbitos nacional e internacional, como la crisis financiera mundial de 2008 y el aumento de los niveles de violencia asociados al narcotráfico, confirmó que la modernización administrativa, como estrategia gubernamental, no fue un tema importante en la agenda pública, ni siquiera una asignatura pendiente. Habría que mencionar que las reformas administrativas que incluyen elementos de NGP en América Latina no han sido coherentes, ni son por sí mismas un motor de cambio. En la agenda gubernamental mexicana, se promueve el uso de la réplica de experiencias exitosas o "buenas prácticas" como instrumento de apoyo a la toma de decisiones. ${ }^{1}$ Esto podría explicar en alguna medida, las estrategias erráticas de los gobiernos panistas.

Sin embargo, si bien es posible afirmar que existieron diferencias importantes en la forma y contenido de las reformas administrativas impulsadas por los gobiernos de Vicente Fox y de

${ }^{1}$ Netzahualcóyotl Ornelas y Héctor Ruíz López, "Problemas de la implementación de la Nueva Gestión Pública en América Latina”, Fundación José Ortega y Gasset, p. 22. 
Felipe Calderón, también es cierto que contienen preocupaciones compartidas, como el cambio democrático y el énfasis en las medidas administrativas de carácter gerencial, lo que permite trazar algunos paralelismos a lo largo del periodo en el que estuvieron en el poder gobiernos emanados del Partido Acción Nacional, el PAN.

En este artículo se presenta un análisis del proceso de modernización administrativa del gobierno de Felipe Calderón, observándolo a la luz de su particular entorno político, además de intentar compararlo, en algunos puntos, con aquellas acciones que se llevaron a cabo en el gobierno de Vicente Fox. En general, se propone en este artículo un análisis sobre las acciones en materia de reforma administrativa en el gobierno de Felipe Calderón, analizando también las estrategias particulares que se pusieron en marcha y los resultados que se ofrecieron, sobre todo hacia finales del sexenio. La premisa principal es que los factores contextuales políticos y económicos que se presentaron en el momento en el que el gobierno de Felipe Calderón llega al poder y durante su administración, condicionaron las estrategias de modernización administrativa y limitaron el esfuerzo que podría haberlas ayudado en términos de su utilidad. Esto dio lugar a cambios aislados en la administración, como la insistencia en la adopción de herramientas gerenciales, más que en un proceso de reforma integral que buscara fortalecer institucionalmente a la administración pública, al menos a nivel federal.

El presente artículo se divide en dos secciones. La primera analiza las ideas teóricas de la modernización administrativa como proceso transformador de las relaciones de poder y de esquemas de incentivos dentro del aparato burocrático, así como el sincretismo entre la NGP y el paradigma burocrático weberiano, para explicar cómo la adopción de herramientas gerenciales no es antagónica con una burocracia de corte clásico. En la segunda sección se exponen las medidas específicas del proyecto modernizador del gobierno de Felipe Calderón. Éste fue un proceso que desde que inició la administración estuvo limitado por prioridades que no cambiarían durante el sexenio, como la austeridad y la atención a otros temas a los que se les otorgó una evidente prioridad, como el de la inseguridad derivada del aumento del crimen organizado. 


\section{El CAMBio POLÍTICO Y LA MODERNIZACióN} adMinistrativa EN MÉXico

Como punto de partida de este análisis, es necesario buscar líneas explicativas para entender los esfuerzos de modernización administrativa durante el gobierno de Felipe Calderón, en un contexto amplio, como parte de un proceso de mayor envergadura relacionado con la transformación política del Estado y su relación con los ciudadanos. En particular, es pertinente poner atención a la manera en la que la reforma administrativa, como estrategia gubernamental, se enfoca en promover cambios dentro del gobierno y así favorecer nuevas conductas, intereses, actores y, en consecuencia, nuevos equilibrios de poder. En efecto, como se discutirá más adelante, los proyectos y resultados en materia de cambio administrativo propuestos por el gobierno de Felipe Calderón (2006-2012), y los que también se impulsaron por la administración de Vicente Fox (2000-2006), formaron parte, en una buena proporción, de lo que intentó ser una estrategia de renovación política después de casi setenta años de ininterrumpido dominio de los gobiernos provenientes del Partido Revolucionario Institucional, el PRI.

Es necesario señalar que el supuesto básico de este análisis sobre modernización administrativa es que "los cambios administrativos significativos redistribuyen los recursos de poder" y, en particular, que este tipo de reformas implican un cambio sensible del equilibrio entre los funcionarios electos y las burocracias. ${ }^{2}$ Es importante tomar en cuenta que la administración pública mantuvo poca o nula autonomía respecto de la política, dado el largo dominio del PRI en el ejercicio del gobierno y sumado a las características que ese partido y a los propios mecanismos con los que contaba el poder ejecutivo que el propio sistema político mexicano

2 "la experiencia mexicana resalta el hecho de que, en un contexto de desarrollo democrático limitado (como en México anterior a las elecciones de 2000), las reformas administrativas están inevitablemente vinculadas a cambios políticos fundamentales", David Arellano Gault y Juan Pablo Guerrero Amparán, "Stalled Administrative Reforms of the Mexican State", en Ben Schneider y Blanca Heredia (coords.), Reinventing Leviathan: The Politics of Administrative Reform in Development Countries, North-South Carolina Press, 2003, p. 151. 
le otorgaba. ${ }^{3}$ Este señalamiento es especialmente relevante puesto que las transformaciones administrativas impulsadas por los gobiernos del PAN tendieron a tomar como base, en la mayoría de los casos, los preceptos de la NGP, la cual tiene, como uno de sus enunciados normativos principales, que la administración pública debe gozar de un relativo grado de neutralidad e independencia frente al poder político. ${ }^{4} \mathrm{Al}$ respecto, es conveniente hacer una muy breve reflexión sobre la NGP y la administración pública para poder avanzar en la explicación.

Para centrar la discusión en las características más importantes de la NGP como una tendencia de las últimas décadas para modernizar las administraciones públicas, se ha insistido en que no es, en esencia, una opción radicalmente distinta para transformar la administración pública, ni un corpus integrado de teorías y prácticas administrativas, sino que más bien se trata de una serie de recomendaciones formuladas como una suerte de reacción frente a los problemas gubernamentales a la luz de las nuevas posibilidades sociales y tecnológicas que caracterizan al siglo xxi. ${ }^{5}$ En este sentido, se ha entendido a la NGP como un conjunto de técnicas para solucionar problemas propios de la administración privada, aplicadas a las agencias de la administración pública. Así, las características esenciales de esta corriente constituyen también nuevas estrategias de gestión. ${ }^{6}$

Lo destacable de las propuestas de la NGP es subrayar el hecho de que el uso de las nuevas tecnologías de la información, así como el fortalecimiento de prácticas como la gestión basada en resultados

${ }^{3}$ David Arellano Gault, "Nueva Gestión Pública: ¿el meteorito que mató al dinosaurio? Lecciones para la reforma administrativa en países como México", Revista del Centro Latinoamericano de Administración para el Desarrollo, núm. 23, 2002, p. 12.

${ }^{4}$ Se ha considerado que la NGP condensa al menos cuatro características fundamentales, por ejemplo, en el servicio de carrera: anonimato, permanencia, mérito y neutralidad política. Andrew Stark, "What Is the New Public Management", Journal of Public Administration Research and Theory: J-PART, vol. 11, núm. 1, 2002, p. 140.

${ }^{5}$ David Arellano Gault, op. cit., p. 1.

${ }^{6}$ Stephen Page, "What's New about the New Public Management? Administrative Change in the Human Services", Public Administration Review, vol. 65, núm. 6, 2005 , p. 714 . 
y la medición de impacto, entre otras, no necesariamente se oponen a la lógica administrativa weberiana. Por el contrario, incluso, pueden llegar a fortalecerla y hasta complementarla. ${ }^{7}$ De ahí que resulte importante encontrar las coincidencias entre una propuesta como la NGP y la lógica burocrática tradicional, puesto que el proceso de modernización debe hacer frente a decisiones pospuestas como pueden ser, por ejemplo, la necesidad de profesionalizar la función pública, además de seguir presentes déficits en el desempeño tanto institucional como en el de los funcionarios que integran la administración pública.

También es importante subrayar que las reformas administrativas ocurridas en México durante las últimas décadas no han sido, como se ha querido pensar en ocasiones, una expresión más de la oleada internacional de reformas gerencialistas que predominaron en la década de $1990,{ }^{8}$ sino que sus posibilidades y limitaciones estuvieron directamente vinculadas con procesos internos de liberalización económica y democratización política, ${ }^{9}$ aunque se hayan atendido las recomendaciones de organismos internacionales, como claramente ocurrió en el caso del tema de la transparencia.

Como ya se ha apuntado aquí, las reformas administrativas recientes han sido, en la mayoría de los casos, esfuerzos más o menos superficiales de reorganización interna que no han afectado la naturaleza centralizada y jerárquica del sector público, ni tampoco han aumentado la autonomía de las agencias públicas o han logrado introducir mecanismos de mercado en la administración pública en su conjunto. Lo importante aquí es subrayar que las reformas administrativas en México inspiradas en la NGP no han sido estrategias premeditadas de innovación, sino resultado de decisiones incrementales y contingentes, impulsadas por

${ }^{7}$ David Arellano, art. cit., p. 1.

${ }^{8}$ Véase Donald E. Klinger, “'South of the Border': Progress and Problems in Implementing New Public Management Reforms in Mexico Today”, The American Review of Public Administration, núm. 30, 2000.

${ }^{9}$ Guillermo M. Cejudo, "Explaining Change in the Mexican Public Sector: the Limits of the New Public Management", International Review of Administrative Sciences, vol. 74, 2008, pp. 111-127. 
decisiones que implicaban algún grado de intención de cambio, ${ }^{10}$ que podrían materializarse desde el programa de reducción gubernamental de la década de 1980, el incremento de la participación de la sociedad civil y de los medios de comunicación, o la creciente injerencia del Congreso en asuntos públicos; sin olvidar los efectos de la más reciente crisis financiera, que tuvo consecuencias relevantes para acabar restándole importancia a estas propuestas. $^{11}$

En este trabajo se intenta entender a las reformas administrativas como interacciones de poder en las que participan tanto políticos y servidores públicos de diversos niveles, como los medios de comunicación, académicos, legisladores y los propios ciudadanos. ${ }^{12}$ La reforma de la carrera burocrática emprendida en el gobierno del presidente Vicente Fox, por ejemplo, es una modificación que, más allá de su importancia relativa, implica una transformación de una de las prerrogativas históricamente más importantes del partido en el poder; esto es, la capacidad de premiar y castigar mediante la asignación de puestos públicos. En 2012, sin embargo, la reforma al servicio profesional de carrera de la APF modificó los términos en que había sido diseñado ese modelo de profesionalización, de suerte tal que se eliminó la posibilidad de que los servidores de carrera pudieran acceder a los puestos de director general, director general adjunto y homólogos. Esta decisión debilitó al servicio de carrera, puesto que el escalafón resultaba un mecanismo menos atractivo como generador de incentivos para los servidores públicos profesionalizados.

La dimensión temporal es otra de las claves importantes para el análisis. Como en cualquier otro intercambio político, el relativo

${ }^{10}$ Véase Mauricio Dussauge, "Paradoxes of Public Sector Reform: The Mexican Experience (2000-2007)", International Public Management Review, vol. 9, núm. 1, 2008, p. 122.

11 Ibid., p. 123.

12 Véase: Mauricio Dussauge Laguna, "La literatura comparada sobre reformas administrativas”, Gestión y Política Pública, vol. 18, núm. 2, 2008, p. 450; Guy Peters, The Future of Governing, Lawrence, University of Kansas Press, 2001; y Christopher Hood y Martin Lodge, The Politics of Public Service Bargains, Oxford, Oxford University Press 2006. 
éxito o fracaso de las reformas administrativas de estos periodos dependió de la capacidad de los gobiernos para poner en marcha varias modificaciones normativas y organizacionales a lo largo de distintos periodos en el tiempo o incluso de manera simultánea, situación a la que se enfrentó particularmente el gobierno de Vicente Fox. En muchos casos, fue precisamente la incapacidad de prever los efectos, en ocasiones perversos, de cada uno de los elementos de las reformas administrativas sobre el resto, lo que conllevó a efectos negativos en el resultado global de la reforma, además de esperar profundas transformaciones en un horizonte muy corto en el tiempo.

En este sentido, el reto de mayor complejidad que caracterizó las reformas del periodo analizado fue, sin lugar a dudas, la creación de un grupo de ganadores definido y sostenido fuera y dentro de los aparatos de gobierno, con capacidad de afrontar los altos costos de la reforma, así como la oposición de aquellos que resultaran afectados por la misma, como podría haber sido el de los propios funcionarios y grupos dentro de la sociedad que habían presionado para contar, por ejemplo, con una administración más transparente. Para el caso de estas reformas se requería, entonces, construir nuevos equilibrios. Dicha tarea implicaba necesariamente una definitiva capacidad de liderazgo y de estrategia que lograra la distribución, entre distintos grupos de actores, de los beneficios, costos y riesgos. Esa estrategia no estuvo presente en los esfuerzos de reforma.

El liderazgo en la bibliografía sobre administración pública es una de las variables que puede ayudar a explicar el éxito de los cambios administrativos. Desde el pensamiento conductista se argumenta que un ejecutivo eficaz debe negociar algunas decisiones en un contexto de limitaciones como la complejidad organizacional, el tiempo insuficiente y la escasa y deficiente información. Para atender las dos primeras limitaciones, los líderes deben delegar autoridad y respetar roles. ${ }^{13}$ Para contar con información de

${ }^{13}$ Stephen P. Mumme y Debra J. Little, "Leadership, Politics, and Administrative Reform at the United States Section of the International Boundary and Water Commission, United States and Mexico", The Social Science Journal, vol. 47, núm. 2, 2010, p. 254. 
calidad, los líderes deben ser buenos comunicadores y deben evitar el envío de señales que disminuyan la efectividad del cambio hacia sus subordinados. Un ejemplo contrario de lo anterior se encuentra en la presentación del Programa Especial de Mejoramiento de la Gestión, cuando Felipe Calderón desvió la atención del programa por un concurso para identificar los trámites más inútiles. En efecto, un líder que busque la transformación debe ser capaz no sólo de articular una visión de cambio, sino de comprometer a otros en ese particular proceso. Al gobierno de Vicente Fox se le cuestionó la capacidad de impulsar las llamadas reformas estructurales, que encontraron en el Congreso un fuerte contrincante, el Partido Revolucionario Institucional. Felipe Calderón no pudo impulsar reformas de mayor calado, como la energética, puesto que tampoco contó con apoyo suficiente en los partidos de oposición. La incapacidad del ejecutivo federal para crear acuerdos dentro del poder legislativo se fue convirtiendo en un rasgo distintivo de los gobiernos panistas.

Además de un liderazgo cuestionado en las dos administraciones panistas, quizá acentuado en el gobierno de Felipe Calderón por la crisis de legitimidad que ocasionaron los comicios electorales de 2006, los procesos se complicaron aún más. A la dificultad natural de la resistencia frente a procesos de cambio habría que sumar que los modelos de reforma introducidos en estos últimos años se pusieron en marcha respondiendo a lógicas burocráticas distintas y hasta opuestas, sin reflexionar en el hecho de que pudieran haberse entendido como propuestas que podrían haberse fortalecido entre sí o, al menos, haberse entendido como complementarias. Se introdujeron como propuestas distintas debido, entre otras cosas, a que se promovieron por instancias burocráticas también distintas, las cuales efectivamente respondían a lógicas burocráticas diversas y hasta antagónicas. Como ejemplo claro de esto, es posible señalar por un lado las reformas de clásico cuño weberiano, como la profesionalización del servicio burocrático, y, por el otro, reformas más típicamente gerenciales, como la evaluación del desempeño a partir de resultados o la desregulación de servicios y trámites. Esta superposición de modelos de reforma implicó serias dificultades en el momento de poner en práctica las 
distintas medidas de mejora gubernamental, al punto de que, en algunos casos, se obstaculizaron unas a otras. ${ }^{14}$ La dificultad de conciliar modelos de reforma administrativa, entonces, se sumó a los contratiempos generados por la oposición no sólo de cuerpos técnicos, sino de proyectos originados en dependencias distintas. Esta situación que se tradujo en una suerte de competencia de modelos administrativos, pero también en un claro enfrentamiento entre agencias gubernamentales.

\section{AUSTERIDAD O REFORMA}

Los cambios administrativos pensados desde el gobierno federal se consolidaron en el Plan Nacional de Desarrollo 2007-2012, documento rector del sexenio de Felipe Calderón. Además, se aprobó un nuevo Reglamento de la Ley del Servicio Profesional de Carrera (Diario Oficial de la Federación [DOF], junio de 2007). Como resultado de la aprobación de la Ley Federal de Presupuesto y Responsabilidad Hacendaria (DOF, octubre de 2007), se introdujeron otros instrumentos como el Presupuesto basado en Resultados (PbR), que incluyó los mecanismos de evaluación y desempeño del gobierno federal materializados en el Sistema de Evaluación del Desempeño (SED); también se propuso el Programa Especial de Mejora de la Gestión de la Administración Pública Federal 2008-2012, que se puso en marcha en octubre de 2008, de manera simultánea con el concurso del trámite inútil.

A pesar de estos resultados, la modernización administrativa no contó desde sus inicios con la importancia concedida en el gobierno anterior. A lo largo del sexenio tampoco logró colocarse como prioridad en la agenda del gobierno federal. Esto se debe entre otras cosas a que fue un periodo marcado, en un principio, por una legitimidad cuestionada y, posteriormente, por una de las

${ }^{14}$ Véase Juan Pablo Guerrero, "La reforma a la administración pública mexicana en el nuevo régimen político. ¿Por dónde empezar? Ideas para la creación de un gobierno transparente, responsable y cercano a la ciudadanía”, México, Centro de Investigación y Docencia Económicas, Cuadernos de Trabajo, 2001. 
crisis económicas y financieras más adversas para la economía de México y del mundo. Adicionalmente, otros asuntos críticos en el país también requirieron de la mayor atención del gobierno federal, como es el caso del crimen organizado y el tráfico de drogas, que provocaron niveles de inseguridad hasta entonces desconocidos en el país. La prioridad en la agenda del gobierno de Felipe Calderón estuvo en la puesta en marcha de una política de seguridad que dio pie a un combate frontal entre las instituciones de seguridad del Estado y los grupos criminales.

Si bien es cierto que la modernización administrativa no recibió el mismo impulso que en la administración anterior, y que la crisis financiera afectó la consecución de los objetivos previstos en la agenda, se impulsaron algunos temas incluidos en el Plan Nacional de Desarrollo y en menor medida en el Programa Especial de Mejora de la Gestión. Se intentó fortalecer al sector público, sobre todo por medio de prácticas como el presupuesto basado en resultados; sin embargo, el énfasis en el carácter presupuestal de estas iniciativas hizo patente el hecho de que se carecía de una política más amplia de fortalecimiento institucional e innovación administrativa. Estas propuestas, al no formar parte de un plan más amplio o simplemente coherente, muestran la naturaleza de las reformas neogerenciales en los gobiernos mexicanos. Es decir, no son modelos que integren un solo paradigma administrativo, más bien los cambios administrativos van a la zaga del contexto político específico. En el caso del gobierno de Felipe Calderón, la poca atención a la reforma administrativa en la agenda federal se tradujo en acciones que dieron como resultado respuestas aisladas y hasta erráticas, debido a que carecieron de una estrategia que definiera objetivos, fines y mecanismos para el cambio.

\section{La politica de austeridad}

Durante los primeros días del gobierno del presidente Felipe Calderón en el mes de diciembre de 2006, se lanzó una primera iniciativa para realizar a cabo cambios en la administración pública federal: el llamado decreto de austeridad, que consistió, en la reducción de 
salarios en una proporción de $10 \%$ para los altos funcionarios. Esta medida se tomó también en el caso del salario del propio presidente, los secretarios y subsecretarios, directores y encargados de agencias federales; de igual manera, se redujo el presupuesto de las agencias federales en una proporción de $5 \%$, excepto en aquellas vinculadas con tareas de seguridad. ${ }^{15}$

Adicionalmente, se buscó la reducción de gastos operativos y de nómina, por ejemplo, mediante la disminución de las membrecías de organismos internacionales a las que estaban afiliadas distintas secretarías, así como en gastos de oficina, muebles y servicios de información y tecnología. El objetivo final del decreto fue lograr un ahorro de 25500 millones de pesos que serían destinados al financiamiento de programas sociales para combatir la pobreza.

Si bien el objetivo del decreto fue que los fondos recabados se destinaran a aumentar el financiamiento de los programas de combate a la pobreza en México, con el paso del tiempo, y quizá como efecto de un panorama económico y fiscal cada vez más adverso, la estrategia de austeridad pareció perpetuarse, opacando, en la práctica, las medidas que de manera específica referían a cambios administrativos. De esta manera, la primera nota del nuevo gobierno estuvo destinada a tomar distancia con lo que había hecho el gobierno anterior, y a no continuar ni profundizar las ambiciosas medidas de reforma del sexenio previo. La consigna era dar vuelta a la página y proponer un proyecto distinto.

Esta primera acción del gobierno del presidente Felipe Calderón materializada en el decreto de austeridad se interpretó en varios sentidos, aunque cabe señalar que no se percibió, en general, como una medida destinada a lograr efectos importantes en términos de modernización administrativa. De hecho, se resaltó el énfasis de que la medida pareció no tener motivaciones primordialmente administrativas, sino políticas. Esta decisión se enmarcó en la crisis de legitimidad derivada de los polémicos resultados

${ }^{15}$ Presidencia de la República, "Decreto que establece las medidas de austeridad y reducción del gasto en la administración pública federal”, Diario Oficial de la Federación, 4 de diciembre de 2006, http://www.firco.gob.mx/firco/docs/DecretoDeAusteridad.pdf 
de las elecciones presidenciales del 2006. Al respecto, Mauricio Dussauge señala que:

algunos de los contenidos principales del decreto [de austeridad] eran notablemente similares a los promovidos por otro excandidato presidencial [Andrés Manuel López Obrador], quien anunció que, en el caso de ganar la presidencia, recortaría el salario de los oficiales del gobierno, reduciría el gasto gubernamental e incrementaría los fondos para programas sociales. En este sentido, el decreto de austeridad parece más una política orientada a reducir la polarización política resultado del proceso electoral del año 2006, o un esfuerzo para acercar el gobierno del presidente Calderón a grupos de políticos de izquierda, o ambos; más que un conjunto de medidas claras para lograr el ahorro del dinero de los contribuyentes, mejorar el desempeño burocrático u obtener recursos adicionales para el financiamiento de programas de política social. ${ }^{16}$

La reducción de costos, particularmente los asociados al número de empleados y al nivel de salarios, se transformó de manera casi natural en una de las más importantes prioridades. ${ }^{17}$ Este fue claramente el caso de la estrategia de austeridad hacia el final de su primer trienio de gobierno. Aprovechando, como es común en estas situaciones, el consenso que se genera dentro y fuera del gobierno alrededor de la idea de reducir el tamaño del Estado y sus costos, se amplía el margen de maniobra de los reformadores para emprender otras acciones.

Sin embargo, la política de austeridad puesta en marcha por el presidente Felipe Calderón queda como una muestra más de la incapacidad de crear una fuente de apoyo alrededor de la agenda de reforma lo suficientemente amplia como para sobrellevar periodos críticos, como el que representó la crisis económica internacional. Así, el énfasis en la reducción de costos de operación y nómina no fue en ningún caso una alternativa seria

16 Mauricio Dussauge Laguna, "Paradoxes of Public Sector Reform: The Mexican Experience (2000-2007)”, p. 69.

${ }^{17}$ Ibid., p. 16. 
de mejora gubernamental, sino un conjunto de medidas destinadas a ahorrar recursos en el marco de una severa reducción de los recursos fiscales y económicos del gobierno. Quizá una de las decisiones más sintomáticas en este sentido fue la propuesta, finalmente no realizada en ese momento, de eliminar la Secretaría de la Función Pública, que incluía entre sus tareas principales la continuidad y mejora de la gestión del gobierno mexicano, sin que en dicha propuesta apareciera debidamente reasignada esta función a ninguna otra dependencia. Cabe señalar que esta dependencia del gobierno federal también se intentó desaparecer durante el primero año de la administración de Enrique Peña Nieto, quedando hasta ahora en una crítica situación de indefinición, en espera de que se avance en la idea de la Comisión Anticorrupción, que también está detenida (al momento de esta investigación).

Las acciones de modernización administrativa durante el gobierno de Calderón fueron más bien erráticas y escasas, sin poder enmarcarse en una estrategia de mayor envergadura. A ello hay que sumar el hecho de que, como se ha afirmado, la política de austeridad surgió impulsada por motivos políticos, en una coyuntura en la que el gobierno de la república buscó distender el conflicto en medio del que se había asumido la presidencia. Una vez que esas condiciones se estabilizaron, la política de austeridad se prolongó, pero por otro motivo: la disminución de recursos del gobierno. Es claro que, habiéndose podido constituir la política de austeridad en un medio para la reforma administrativa, el gobierno federal abdicó de esa posibilidad para, en su lugar, usar esa política como una débil tabla de salvavidas económico.

\section{El Plan Nacional de Desarrollo y la modernización administrativa}

En el Plan Nacional de Desarrollo 2007-2012 (PND) se establecieron los ejes de acción que guiarían las estrategias de modernización administrativa; no obstante, desde antes de su publicación se empezaron a delimitar algunos temas que el gobierno impulsaría como reforma al sector público. 
Durante los primeros cien días de gobierno las únicas acciones encaminadas a la modernización fueron una "reducción del gasto burocrático" y "mayor rendición de cuentas". ${ }^{18}$ Se puede observar que desde el inicio de la administración existía la intención de reducir el gasto, intención que más tarde se convertiría en una acción concreta incluida en el mencionado decreto de austeridad del gobierno. En el mismo sentido, la transparencia y rendición de cuentas consumieron cierta atención durante esta administración, puesto que se reformó el artículo sexto constitucional en junio de 2007 y, más tarde, en diciembre de 2008, se puso en marcha el Programa Nacional de Rendición de Cuentas, Transparencia y Combate a la Corrupción como una acción enmarcada dentro del PND (2007-2012), que debía servir, en palabras del secretario de la Función Pública, para "promover estrategias con enfoque preventivo para evitar la corrupción". ${ }^{19}$

Hay que insistir en que el contexto político no resultaba favorable para proponer cambios de gran calado, debido, entre otras cosas, a la debilidad con la que el gobierno inicia su mandato, a causa de problemas de legitimidad derivados de la elección, además de que tomó más tiempo del necesario la promulgación del PND. Esto confirma la idea de que no existía un proyecto integral de reforma administrativa y que las medidas tomadas más adelante, como la adopción de herramientas gerenciales, fueron aisladas.

No obstante, el PND sirvió como documento guía para encaminar los cambios en la administración. Éste incluyó cinco ejes de acción: 1) Estado de derecho y seguridad, 2) economía competitiva y generación de empleos, 3) igualdad de oportunidades, 4) sustentabilidad ambiental y 5) democracia efectiva y política exterior responsable. El tema del mejoramiento de la administración pública federal se ubicó en el eje de democracia efectiva y política exterior responsable. El tema destacó dos puntos en particular, la eficacia y eficiencia gubernamental y la transparencia y rendición

18 En el "Informe de los Primeros Cien Días del Gobierno de Felipe Calderón”, se establecen esas acciones numeradas como las 23 y 91.

19 Palabras del secretario de la Función Pública, C. P. Salvador Vega Casillas, en la presentación del Programa Nacional de Rendición de Cuentas, Transparencia y Combate a la Corrupción 2008-2012. 
de cuentas. Las dependencias responsables de estas acciones fueron la Secretaría de la Función Pública (SFP) y la Secretaría de Hacienda y Crédito Público (SHCP), por momentos de manera individual y en otros intentando establecer mecanismos de coordinación, cuyos resultados no fueron los esperados.

Dentro de las estrategias establecidas en el PND, ${ }^{20}$ en la vertiente de "eficiencia y eficacia gubernamental" se incluyeron los objetivos de: 1) elevar los estándares de eficiencia y eficacia gubernamental; 2) hacer más eficiente la operación y el gasto de las dependencias y entidades; 3) profesionalizar el servicio público buscando mejorar el rendimiento de las estructuras orgánicas de la Administración Pública Federal (APF); 4) adoptar un modelo de diseño del Presupuesto basado en Resultados (PbR) que facilitara la rendición de cuentas y generara los incentivos de la APF; 5) evaluar el desempeño de los programas de gobierno y su impacto en la población, mediante un Sistema de Evaluación del Desempeño (SED); 6) coordinar y establecer mecanismos para la transparencia y rendición de cuentas de los gobiernos estatales y municipales en el ejercicio de los recursos federales como política pública permanente en los niveles de gobierno. A juzgar por los resultados de las estrategias descritas, resulta evidente que la corresponsabilidad que debió existir entre la SFP y la SHCP, puesto que se incluían acciones que debían haber realizado ambas dependencias, no lllegó a establecerse de manera sistemática y menos aún coordinada. Lo que está documentado remite a un pobre desempeño de las dos dependencias a las que se les encargó coordinarse para impulsar acciones en materia de modernización administrativa. ${ }^{21}$

${ }^{20}$ El PND establece el compromiso de mejorar la eficacia y eficiencia gubernamental, mediante estrategias que permitan el uso honesto, inteligente y austero de los recursos públicos, así como la aplicación de acciones de sistematización, digitalización y profesionalización de los servicios públicos para incrementar la calidad en la formulación de procesos, trámites y prestaciones de servicios públi$\cos$ (PND, 2007-2012).

${ }^{21}$ En la vertiente de "transparencia y rendición de cuentas" existen acciones muy concretas que deberá realizar la administración pública federal para "garantizar la transparencia, la rendición de cuentas, el acceso a la información y la protección de los datos personales en todos los ámbitos de gobierno”. Resulta 
Del PND se desprendió una serie de mecanismos para la modernización administrativa. El primero de ellos fue el Presupuesto basado en Resultados (PbR), con el que se pretendía evaluar el impacto del gasto mediante procesos e instrumentos que permitían que las decisiones involucradas en el presupuesto incorporaran consideraciones sobre los resultados del uso de los recursos públicos. El segundo instrumento fue el SED, que permitiría evaluar los logros de los programas gubernamentales y las políticas públicas, bajo los principios de verificación del grado de cumplimiento de las metas y objetivos, con base en los indicadores estratégicos y de gestión. Adicionalmente, durante este periodo se contó con el Programa Especial de Mejoramiento de la Gestión 2008-2012 (PMG), para evaluar el desempeño de las instituciones y los funcionarios públicos. ${ }^{22}$

\section{El Servicio Profesional de Carrera}

El control burocrático es una de las primeras preocupaciones de los gobiernos democráticos o de aquellos que intentan transitar hacia ese sistema. La discusión académica señala que los gobiernos con democracia representativa buscan asegurar que las decisiones de política coincidan con los intereses o preferencias de los ciudadanos por medio de, al menos, dos instrumentos: procedimientos administrativos o de la "supervisión"; es decir, el seguimiento, la gratificación o sanción al comportamiento burocrático a través de un servicio civil. La implementación de un servicio civil en México se pudo llevar a cabo después de la alternancia partidista.

\footnotetext{
significativo que en los programas derivados del PND, aparezca sólo un listado, y dentro de él, el mencionado programa, de Transparencia y Rendición de Cuentas y Combate a la Corrupción, aprobado a finales de 2008. Este programa quedó a cargo de la SFP.

${ }^{22}$ Véase Salvador Vega Casillas, "El Programa de Mejora de la Gestión”, Conferencia Internacional sobre Presupuesto Basado en Resultados, ciudad de México, 2008; también, Vega Casillas, "La modernización del gobierno mexicano a partir de la mejora de la gestión: entrevista al Secretario de la Función Pública”. Entrevista realizada por José Sosa”, RAP, México, INAP, vol. 43, núm. 1, 2008.
} 
La reforma del servicio profesional que englobara a la APF centralizada se aprobó en 2003 por el Congreso de la Unión con ningún voto en contra. ${ }^{23}$

Hasta antes de esa fecha existieron esfuerzos aislados, y no necesariamente de tipo normativo, que intentaron promover un sistema que regulara el ingreso, permanencia y promoción a los puestos dentro de la APF. Por ejemplo, en 1982 se crea la Coordinación para la Modernización de la Administración Pública, que dependía de la Subsecretaria de Control Presupuestal y Contabilidad de la Secretaría de Programación y Presupuesto. Dentro de esta secretaría se crea también la Dirección del Servicio Civil de Carrera, responsable de normar y coordinar el servicio civil sólo para esa dependencia. Con el Programa de Modernización de la Administración Pública 1995-2000, se propuso un servicio profesional que garantizara la adecuada selección y el desarrollo profesional de los servidores públicos, pero el proyecto no logró consolidarse. ${ }^{24}$ Existen, sin embargo, algunas experiencias concretas de profesionalización de los servidores públicos, como es el caso de la Secretaría de las Relaciones Exteriores (el Servicio Exterior Mexicano), del Instituto Federal Electoral (el Servicio Profesional Electoral) y la Procuraduría Agraria (Servicio Profesional Agrario). ${ }^{25}$

No fue sino hasta 2003 que se publica la Ley del Servicio Profesional de Carrera de la Administración Pública Federal (LSPCAPF) con el objetivo de instaurar un sistema basado en el mérito como un esfuerzo encaminado a la profesionalización de los servidores públicos. ${ }^{26}$ Uno de los antecedentes del Servicio Profesional

${ }^{23}$ José Luis Méndez, "El servicio profesional de carrera de la Administración Pública Federal”, en José Luis Méndez (coord.), Los grandes problemas de México, XIII, Políticas Públicas, México, El Colegio de México, 2010, p. 184.

${ }^{24}$ Aída Alicia Lugo Dávila, "La función pública en México: de la selección y profesionalización en el servicio profesional de carrera", tesis de doctorado, Madrid, Universidad Complutense, 2013, p. 62.

${ }^{25}$ Para un recuento extensivo sobre los esfuerzos para implantar un servicio profesional véase María del Carmen Pardo, "El Servicio Profesional de Carrera en México: de la tradición al cambio”, Foro Internacional, vol. 45, núm. 4, 2005, pp. 599-634.

${ }^{26}$ Rafael Martínez Puón, "La nueva fisonomía del servicio profesional de carrera en México”, Reforma y Democracia (Revista del CLAD), núm. 43, 2009, p. 1. 
fue el Programa Nacional de Combate a la Corrupción y Fomento a la Transparencia y el Desarrollo Administrativo del gobierno de Vicente Fox, que incluyó como una de las metas la expedición de una ley del servicio civil. Sin embargo, desde un inicio la propuesta de servicio civil encontró mucha resistencia, incluso dentro de los mismos funcionarios que acaban de llegar. ${ }^{27}$

La puesta en marcha del SPC no estuvo exenta de dificultades: a) la Secretaría de la Función Pública se transformó en una dependencia centralizadora, lo que dificultó su operación; $b$ ) no todos los subsistemas del servicio profesional se lograron poner en marcha de forma oportuna. ${ }^{28}$ A pesar del establecimiento de un sistema de ingreso basado en el mérito, se utilizó de manera excesiva el artículo 34 de la LSPCAPF. ${ }^{29}$ Esto apunta a que en el gobierno continuó con la vieja práctica de utilizar sus prerrogativas para "colocar" candidatos en puestos de la APF.

Para dar respuesta a estos problemas, el gobierno de Felipe Calderón publica a finales de 2007 un nuevo reglamento de la LSPCAPF. Sin embargo, de nueva cuenta el uso excesivo del artículo 34 demuestra que el objetivo no era descentralizar funciones, sino reducir el alcance del sistema. ${ }^{30}$ Es importante hacer mención que también se aprobó el Programa para el Servicio Profesional de Carrera en la Administración Pública Federal 2008-2012, con el propósito de "garantizar la operación integral del Sistema, a través de la participación y coordinación de los diferentes actores que intervinieran; resultando obligatorio para las dependencias y órganos administrativos desconcentrados a los que les resultaba aplicable la

${ }^{27}$ José Luis Méndez, art. cit., p. 183.

28 Ibid., p. 187.

${ }^{29}$ Como señala Méndez, este artículo establece una excepción, de extrema necesidad (emergencias), para el ingreso por concurso. "En los últimos tres años del gobierno de Fox hubo 2290 nombramientos por el artículo 34 (1 435 en 2004, 422 en 2005 y 433 en 2006)", "en los últimos tres años del gobierno de Fox hubo 2290 nombramientos por el artículo 34 (1 435 en 2004, 422 en 2005 y 433 en 2006)". Ibid., p. 189.

30 "Cerca de 7000 nombramientos sin concurso del primer año del gobierno de Calderón (julio de 2007-julio de 2008) superaron por más del doble a aquéllos de los últimos tres años del pasado sexenio [de Vicente Fox]", Loc. cit. 
LSPC". ${ }^{31}$ Este programa también se alineó con el PND 2008-2012, al establecerse que la profesionalización a los servidores públicos debía estar orientada a resultados y al fortalecimiento de una cultura de evaluación del desempeño, por medio del ingreso y permanencia en el servicio público basados en el mérito. En este sentido, con la profesionalización de los servidores públicos se buscaba contribuir a la adquisición de herramientas y a lograr un desarrollo profesional para que pudiera implementarse la gestión por resultados, uno de los supuestos ejes de la "modernización administrativa" de Felipe Calderón.

A pesar de esos enunciados, la profesionalización de los servidores públicos por medio este sistema (SPC) tampoco fue una prioridad para el gobierno del presidente Felipe Calderón. Si bien este programa se empezó a elaborar en 2007, no fue sino hasta 2010 que se autoriza su publicación. ${ }^{32} \mathrm{Y}$ es hasta el 30 de julio de 2012, cinco meses antes de que acabara el sexenio, cuando se publica en el Diario Oficial de la Federación. Esto constituye una clara evidencia del poco interés que tuvo el gobierno de Felipe Calderón en este tema.

\section{El Programa Especial de Mejora de la Gestión Pública}

El objetivo del PMG era potenciar una gestión pública orientada hacia resultados vinculándola con la asignación de recursos presupuestarios, con la aplicación de procesos de planeación estratégica, con la responsabilidad por el cumplimiento de las metas programadas mediante la construcción de incentivos y el establecimiento de sistemas de control de gestión interna. Lo anterior tenía el propósito de alcanzar la eficiencia, calidad y transparencia de la gestión pública.

Cabe señalar que se trató de una propuesta de reforma de inspiración fundamentalmente gerencial, es decir, orientada a introducir prácticas del sector privado en la gestión pública; aunque a

${ }^{31}$ DOF, 30/07/2012, "Acuerdo por el que se da a conocer el Programa para el Servicio Profesional de Carrera en la Administración Pública Federal”.

${ }^{32}$ Lugo Dávila, op. cit., p. 186. 
diferencia del programa que puso en marcha el gobierno anterior, las prácticas de la administración privada buscaron introducirse de manera selectiva y parcial. ${ }^{33}$ Los cinco ejes a través de los cuales se organizó el programa subrayaron los elementos más característicos de este tipo de reformas: 1) la orientación a resultados; 2) la flexibilidad, entendida como el reconocimiento de los diversos niveles de desarrollo institucional entre las dependencias públicas; 3) la innovación, 4) la sinergia, es decir, la integración en un mismo "círculo de mejora de la gestión" de los actores y dependencias públicas; y 5) la participación ciudadana, que buscaba integrar las opiniones y necesidades ciudadanas en la mejora del gobierno. ${ }^{34}$ Sin embargo, como ya se ha señalado, ninguna de estas medidas se vinculó con una transformación estructural del funcionamiento de la administración pública federal, por ejemplo, mediante el otorgamiento de autonomía a agencias públicas.

Aunque en el caso del PMG se intentó descentralizar la operación del programa mediante la asignación de responsabilidades a funcionarios en cada una de las dependencias del gobierno, lo cierto es que esa decisión terminó por aumentar el riesgo de que se evadiera el cumplimiento de los controles y metas correspondientes. ${ }^{35}$ La situación fue todavía más adversa si se considera que la adquisición de nuevas habilidades y responsabilidades por parte de los funcionarios no sustituía sino que se sumaba a sus tareas tradicionales, además de no ser recompensada en términos salariales, ni de ningún otro modo. ${ }^{36}$

El PMG constituyó el instrumento específico de mayor alcance para realizar mejoras que orientaran de manera sistemática la gestión de las instituciones públicas para lograr mejores resultados. Para su efectiva instrumentación se requería una implantación adecuada que permitiera dar certidumbre y claridad a las acciones comprometidas en el marco del programa, coordinadas por la SFP. El

${ }^{33}$ Cejudo, art. cit., p. 120.

34 Secretaría de la Función Pública, Programa Especial para la Mejora de la Gestión en la Administración Pública Federal 2008-2012, http://www.funcionpublica.gob.mx/index.php/pmg.html

${ }^{35}$ Dussauge, op. cit., pp. 66-68.

${ }^{36}$ Loc. cit. 
programa, sin embargo, no logró acreditarse como el programa rector en esa materia, entre otras cosas porque la SFP invirtió una parte importante del periodo de gobierno en su reestructuración interna, y, también, porque el día de su anuncio, el propio presidente Calderón ofreció la puesta en marcha del "Concurso para identificar el trámite más inútil”, que como se señaló restó toda la importancia y visibilidad al PMG. El objetivo del concurso era incluir la opinión de la ciudadanía por medio de propuestas para el mejoramiento de trámites en los tres órdenes de gobierno; los ganadores recibieron un premio de entre 100000 y 300000 pesos, dependiendo la categoría. ${ }^{37}$ Sin embargo, esta "ocurrencia" del presidente no estuvo vinculada a un programa integral de reforma administrativa. ${ }^{38}$

La decisión del gobierno de Felipe Calderón de apostar por una reforma de corte gerencial, como la del PMG, tiene una lógica subyacente. Al contrario que las reformas de servicio civil o las reformas de transparencia, las reformas gerenciales parecen tener dos ventajas importantes: 1) el que no afectan el dominio tradicional de la élite política sobre la asignación de puestos públicos; y 2) la reducción de los costos de monitoreo durante su implementación. ${ }^{39}$ En el primer rubro, la reforma del servicio civil de carrera en realidad alcanzó resultados pobres en términos de asegurar la neutralidad política de los funcionarios, de tal manera que las medidas incluidas en el PMG tuvieron muy poco efecto en la alteración de dicho equilibrio. La segunda ventaja surge del hecho de que, en

${ }^{37}$ Secretaría de la Función Pública, "Convocatoria del Concurso para identificar el Trámite más Inútil”, publicado en septiembre de 2008. El premio para la mejor propuesta en la categoría "Nivel Federal" fue de 300000 pesos, y para las categorías "Nivel Estatal” y "Nivel Municipal” el premió constó en 100000 pesos.

38 "En aquella ocasión [en alguna reunión con algunos servidores públicos hace meses] sugería yo que organizáramos un concurso muy especial entre los ciudadanos; y ahora no lo voy a sugerir, le voy a instruir al Secretario de la Función Pública, para que lo haga y lo organice este mismo mes de septiembre", Felipe Calderón Hinojosa, "Discurso del presidente en la presentación del Programa Especial de Mejora de la Gestión 2008-2012”, pronunciado el 9 de septiembre de 2008 en la Ciudad de México, en http://www.funcionpublica.gob.mx/index. $\mathrm{php} / \mathrm{ua} / \mathrm{ssfp} /$ el-tramite-mas-inutil/discurso-del-presidente.html, consultado en noviembre de 2014.

${ }^{39}$ Schneider y Heredia (coords.), op. cit., p. 21. 
su mayor parte, las metas de la reforma gerencial dependen de las presiones competitivas que se asocian con la misma. Hay que decir, sin embargo, que esas ventajas no pudieron aprovecharse debido, entre otras cosas, a que estos cambios no se acompañaron de un esquema de incentivos que hubiera introducido una competencia positiva en materia de mejora en los resultados.

\section{Ley Federal de Presupuesto y Responsabilidad Hacendaria y la evaluación de resultados y el desempeño}

A partir del 2006 y como parte de la estrategia de modernización administrativa del presidente Felipe Calderón, debe observarse, como una decisión importante, la promulgación de la Ley Federal de Presupuesto y Responsabilidad Hacendaria (LFPRH), ${ }^{40}$ que abrogó la Ley Federal de Presupuesto, Contabilidad y Gasto Público Federal y su Reglamento, estableciéndose la evaluación del desempeño con base en indicadores de resultados de manera obligatoria para la totalidad de las instituciones de la APF, (DOF, 1 de octubre 2007).

La LFPRH se ancla en tres pilares: el equilibrio presupuestal, la disciplina fiscal y el cumplimiento de metas. En este último punto se materializa la gestión por resultados y se integra la obligación de identificar los indicadores de medición para que la evaluación se lleve a cabo con mecanismos de evaluación del desempeño en el uso de los recursos públicos. Con base en estos resultados debía facilitarse la toma de decisiones para mejorar el desempeño gubernamental. ${ }^{41}$

40 Publicada en el Diario Oficial de la Federación el 30 de marzo de 2006, para tener efectos a partir del 1 de abril del mismo año. De acuerdo con la sHcP, para la aprobación de la ley el proceso tomó casi dos años y se contó con la participación adicional del Congreso de la Unión, de las entidades federativas a través de diversos foros como la Conferencia Nacional de Gobernadores (Conago), de funcionarios públicos federales y del Instituto de Investigaciones Jurídicas, entre otros.

${ }^{41}$ Adriana Verónica Hinojosa Cruz, "Configuración histórica de la nueva Ley Federal de Presupuesto y Responsabilidad Hacendaria”, XI Congreso Internacional del CLAD sobre la Reforma del Estado y de la Administración Pública, Ciudad de Guatemala, p. 8. 
El objetivo principal al expedir el nuevo ordenamiento fue integrar un orden presupuestario con elementos de certidumbre jurídica, económicos y de transparencia en todo el proceso, así como incorporar un nuevo concepto: el de responsabilidad hacendaria. ${ }^{42}$ Además la Ley estableció un nuevo marco jurídico del proceso presupuestario por el que bimestralmente la SHCP y la SFP verificarían resultados y la ejecución de programas y presupuestos, con base en el SED, puesto en marcha de forma definitiva en el ejercicio 2008. El funcionamiento del SED se vincularía con los indicadores de desempeño contenidos en la estructura programática. Asimismo se estableció que ambas dependencias, SHCP y SFP, podrían suscribir convenios de desempeño, en forma anual o plurianual, a fin de establecer compromisos de resultados y medidas presupuestarias para un ejercicio eficiente, con el propósito de impulsar la utilización del PbR. ${ }^{43}$

La evaluación del desempeño se concibió como una herramienta que aportaría insumos para mejorar la gestión pública al producir información necesaria para identificar y entender las causas de los logros y los fracasos o problemas del desempeño individual y colectivo, dentro de un contexto de planeación estratégica. ${ }^{44}$ Se lo concibió también como un proceso que verifica, mide y evalúa la cobertura de estándares previamente marcados para el cumplimiento de los objetivos y metas institucionales. ${ }^{45}$

42 Ibid., p. 7.

43 SHCP, Sistema de Evaluación del Desempeño, México, 2007, p. 7. La SHCP menciona los avances alcanzados para coadyuvar en el PBR, a partir del año 2006 para lo cual realizó un Plan de Acción para implantar el nuevo esquema: 1) desarrollo del modelo $\mathrm{PbR}, 2$ ) estructuración de un sistema integrado de monitoreo y evaluación, 3) adecuación del proceso presupuestario para incorporar información sobre resultados, 4) fortalecimiento del sistema de incentivos sobre las dependencias y entidades y 5) normas sobre la gestión financiera para facilitar el logro de resultados (p. 10).

${ }^{44}$ Ramón Muñoz, "Innovación Gubernamental”, México, FCE, 2004, p. 67.

${ }^{45}$ Luis E. Villanueva Ángel, "La necesidad de la implantación y reforzamiento de metodologías y mecanismos de evaluación del desempeño del elemento humano en la administración pública mexicana”, VIII Congreso Internacional del CLAD sobre la Reforma del Estado y de la Administración Pública, Panamá. 
Como parte de esta estrategia de modernización administrativa, el PbR se define como el conjunto de procesos e instrumentos que permitirían que las decisiones involucradas en el presupuesto incorporaran consideraciones sobre los resultados obtenidos en la aplicación de los recursos públicos. ${ }^{46}$ Se concibió para facilitar la rendición de cuentas y generar incentivos para que la APF cumpliera con las metas planeadas, evaluara el desempeño de los programas de gobierno y su impacto en la población, bajo principios de transparencia, imparcialidad, eficacia, competitividad y sustentabilidad. ${ }^{47}$ De esta manera, el PbR supuso cuatro etapas para su puesta en marcha: 1) programas y proyectos alineados al PND; 2) definición de objetivos estratégicos, indicadores y metas; 3) seguimiento constante de los recursos enfocados en los resultados; y 4) asignación de recursos en función de la relevancia estratégica y del resultado de las evaluaciones. Adicionalmente, el PbR implicó un nuevo enfoque de gestión pública para incentivar el logro de resultados, por lo que se requería modificar y mejorar de manera estructural las decisiones y las medidas del proceso de planeación, programación, presupuestación y ejercicio del gasto, haciendo énfasis en la evaluación de los programas de gobierno. ${ }^{48}$ El PbR se enfocaría en la definición y establecimiento de los objetivos de manera clara y sencilla y de los resultados que se preveía alcanzar con los programas a los que se les asignaban recursos presupuestarios. ${ }^{49}$

Una vez puesto en marcha el $\mathrm{PbR}$, el siguiente paso consistió en establecer el SED, que se define como el conjunto de elementos metodológicos que permitirían realizar una valoración objetiva del desempeño de los programas, bajo los principios de verificación del grado de cumplimiento de metas y objetivos, con base en

${ }^{46}$ Salvador Vega, "El Programa Especial de Mejora de la Gestión”, p. 7.

47 Salvador Vega, "La modernización del gobierno mexicano a partir de la mejora de la gestión: entrevista al Secretario de la Función Pública. Entrevista realizada por José Sosa”, p. 67.

${ }^{48}$ SHCP, Presupuesto basado en Resultados (PbR) y Sistema de Evaluación del Desempeño (SED). México, Subsecretaría de Egresos, Unidad de Política y Control Presupuestario, 2008, p. 6.

49 Ibid., p. 4. 
indicadores estratégicos y de gestión, para conocer el impacto social y económico de los programas y proyectos. ${ }^{50}$

\section{Sistema de Evaluación del Desempeño (SED)}

En 2007, el SED se puso en marcha junto con los Lineamientos Generales para la Evaluación de los Programas Federales, para unificar las prácticas de evaluación de las dependencias del gobierno federal. Además, un año después la SHCP dio a conocer los Lineamientos Generales para la Programación del Presupuesto Federal para establecer los procedimientos básicos para implementar el SED y el PbR. ${ }^{51}$

El SED contó con dos formas de evaluación para la ejecución de los programas y de los presupuestos. La primera evaluación de programas estuvo destinada a justamente valorar diversos conceptos, con el objetivo de reasignar el presupuesto de los programas con base en sus resultados. ${ }^{52}$ Los conceptos fueron: consistencia y resultados (analiza el diseño y desempeño global de los programas), procesos (analiza mediante trabajo de campo, si se llevaron a cabo los procesos de manera eficaz y eficiente), impacto (identifica el cambio en los indicadores atribuible a la ejecución de los programas) y específica (analiza aspectos o situaciones prioritarias de los programas no comprendidas en los tipos anteriores).

La segunda evaluación analizaría estrategias, políticas y de gestión institucional. El SED realizaría una evaluación de programas, la que se iría operando de forma gradual. En una primera etapa la evaluación del desempeño iba a tomar como base los principales programas por medio de los cuales se ejercería un alto porcentaje del gasto programable, excluyendo servicios personales, aportaciones federales y las asignaciones a los ramos autónomos.

${ }^{50}$ Ibid., p. 21.

${ }^{51}$ Manuel Fernando Castro et al., El Sistema de MẼE en México: un salto del nivel sectorial al nacional, Serie de documentos de trabajo ldce, Banco Mundial, 2009, p. 5.

${ }^{52}$ José Juan Sánchez González, "El cambio institucional en la reforma y modernización administrativa de la administración pública mexicana”, Gestión y Política Pública, vol. 18, núm. 1, 2009, p. 93. 
Esta dinámica traería como resultado que "las visiones eficientistas y de estricto control presupuestal se impondrían como criterios principales de actuación del gobierno, con que se relegaba a un segundo nivel las orientaciones de tipo sectorial y los enfoques que enfatizan la búsqueda de resultados, la satisfacción ciudadana y la puesta en práctica de nuevos modelos de gestión pública”. ${ }^{53}$ Más importante todavía, el mismo Programa de Mejora de la Gestión, plataforma de la innovación administrativa del presidente Felipe Calderón, quedaría severamente afectado, puesto que, por un lado, fue perdiendo fuerza la iniciativa relativa a la implantación del PbR, y el SED se limitó al cumplimiento de metas presupuestales; se olvidaron las cuestiones de fondo relacionadas con el desarrollo institucional del gobierno y con la creación de nuevas capacidades técnicas y humanas. ${ }^{54}$

Por una parte, el rezago en el interés por el desarrollo institucional se debe a dos razones. Primero, la modernización administrativa no ocupó un lugar importante en la agenda gubernamental del gobierno de Felipe Calderón; segundo, la implementación de estas herramientas ha respondido en una buena proporción a recomendaciones realizadas por organizaciones internacionales, como la Organización para la Cooperación y Desarrollo Económicos (OCDE) y el Centro Latinoamericano de Administración para el Desarrollo (CLAD), más que a necesidades de la misma administración federal. ${ }^{55}$ Por la otra parte, la creación de nuevas capacidades se observa en la implementación de la Matriz de Indicadores para Resultados (MIR), herramienta que utiliza la metodología de matriz de marco lógico, cuya definición implica limitaciones muy importantes, que se ilustran en la siguiente sección.

\section{Matriz de Indicadores para Resultados}

Una de las implicaciones derivadas del lanzamiento de los Lineamientos Generales para la Evaluación de los Programas Federales

\footnotetext{
${ }^{53}$ Loc. cit.

${ }^{54}$ Loc. cit.

${ }^{55}$ Loc. cit.
} 
fue la aplicación de herramientas para estandarizar la formulación de indicadores de dichos programas. La primera etapa de implementación de los Lineamientos fue establecer matrices de indicadores basados en la metodología de marco lógico para todos los programas federales con reglas de operación. ${ }^{56}$ La segunda fase consistió en la instauración de los programas anuales de evaluación, impulsada por el Coneval y la SFP en coordinación con las demás dependencias del gobierno federal, para controlar el proceso de evaluación y también "establecer el calendario de ejecución de las evaluaciones de los programas en operación y de los que empezarían su ejecución; asimismo, vincular el calendario de ejecución de las evaluaciones con las actividades de programación y presupuesto, y determinar los tipos de evaluación que se aplicarían mediante un programa integral y gradual, además de articular las evaluaciones en el marco del SED". ${ }^{7}$

Una de las consecuencias surgida de los Lineamientos Generales fue la adopción de la MIR (metodología de marco lógico). Se establecieron Matrices de Indicadores para 389 programas y más de 1620 funcionarios se capacitaron en esta metodología. ${ }^{58} \mathrm{Si}$ bien la elaboración de matrices de indicadores empezó a ser una práctica más o menos generalizada en la administración pública federal, los resultados de su operación han sido muy limitados, porque la herramienta empezó a volverse un fin en sí misma. Más aún, hay casos donde las matrices son poco claras, donde hay deficiencias en la secuencia lógica (tanto vertical como horizontal) y donde la matriz se aplica inadecuadamente a procesos administrativos dentro de las dependencias, más que a un programa público. ${ }^{59}$ Además,

${ }^{56}$ Manuel Fernando Castro et al., op. cit., p. 11.

${ }^{57}$ SHCP, "Programa Anual de Evaluación", http://www.shcp.gob.mx

${ }^{58}$ Manuel Fernando Castro et al., op. cit., p. 12.

${ }^{59}$ Por ejemplo, la Comisión Nacional de Cultura Física y Deporte (Conade), en el anuncio programático de 2012, utilizó la metodología de marco lógico para la construcción de indicadores de "actividades de apoyo administrativo" de la propia Comisión, cuando este tipo de herramientas se utilizan para la planeación y diseño de programas públicas, y en México para la evaluación de los mismos. Consultado en http://sippac2012.sep.gob.mx/anunciopp2012/URs2/L6I/matrizIndicadores.pdf, el 3 de febrero de 2014. 
en algunas dependencias, sino es que en todas, la MIR es un requisito administrativo más a cumplir, en lugar de percibirse como una herramienta que ayude a mejorar la gestión. ${ }^{60}$ Este aspecto de la modernización administrativa llevó a la adopción de herramientas de evaluación por parte de una administración pública con limitado desarrollo en cultura de evaluación y con falta de capacidades para operar estas herramientas.

\section{Conclusiones}

Los alcances del proceso de modernización administrativa del presidente Felipe Calderón se entienden a partir del contexto político que imperó en el momento de la llegada al ejecutivo federal y durante su gobierno. Por un lado, la crisis de legitimidad, resultado de las elecciones de 2006, derivó en el hecho de que la administración entrante buscara acercarse a los grupos políticos de la izquierda y, por tanto, adoptara la política de austeridad administrativa como medida para mitigar la polarización política. Por otro lado, la crisis financiera influyó en la permanencia del decreto de austeridad debido al panorama económico adverso, haciendo que esta política opacara otras medidas de modernización administrativa. Otro factor contextual que explica el poco impulso que se le dio a una reforma integral de la administración fue el aumento en la violencia asociada con el crimen organizado y el narcotráfico. Esta situación desvió la atención y prioridades del gobierno federal, centrando los esfuerzos en la "guerra contra el narcotráfico".

Estos factores contextuales no permitieron que el proceso de modernización ocupara un lugar central en la agenda del presidente Felipe Calderón, ni que fuera un proyecto de largo alcance; más bien fueron medidas aisladas con un enfoque gerencial. De ahí la puesta en marcha de medidas con esta lógica, como el PMG, el PbR y el SED, que no pudieron alterar el equilibrio entre la élite política y la asignación de puestos, ni crear incentivos adecuados 
para ello. Además, son medidas aisladas que responden a circunstancias específicas; por ejemplo, el SED respondió a recomendaciones de organizaciones internacionales, más que a un diagnóstico o requerimientos de la misma administración.

Si bien los procesos de cambio del periodo de Felipe Calderón tuvieron como enfoque central el presupuesto, esto dio lugar a nuevas prácticas dentro de la administración pública, sin que ésta estuviera preparada para ello. La adopción de la MIR obligó a los funcionarios a establecer indicadores de resultados para los programas federales para contar con una herramienta de monitoreo y evaluación. Esto pudo significar sólo un primer paso en el proceso de consolidación de un sistema elaborado para dar seguimiento a sus políticas públicas. No obstante, la adopción de estas herramientas no resultó fácil, puesto que aún no existe una cultura de evaluación consolidada en la administración pública, lo que provoca usos inadecuados y pone en evidencia incapacidades técnicas para aplicar este tipo de herramientas.

La puesta en marcha de medidas enfocadas en el presupuesto parece una tendencia que llegó para quedarse. El Programa para un Gobierno Cercano y Moderno de la nueva administración tiene como objetivos fortalecer el presupuesto basado en resultados de la administración federal. ${ }^{61}$ Esta estrategia pretende vincular el SED con asignaciones presupuestarias, fortalecer el proceso de seguimiento y evaluación al desempeño y mejorar la calidad del gasto. A reserva de un estudio más profundo de los cambios que se pueden generar en materia de modernización administrativa del gobierno del presidente Enrique Peña Nieto, los objetivos planteados en este nuevo programa no arrancan de cero; toman como punto de partida un conjunto de medidas que han estado en operación durante algún tiempo, sin que se hubieran inscrito en una estrategia más integral, a partir de la cual se podían haber esperado mejores resultados de los que se han obtenido hasta ahora, con la puesta en marcha de herramientas inconexas y ajenas a la cultura administrativa que no acaba por cambiar.

${ }^{61}$ Gobierno de la República, Programa para un Gobierno Cercano y Moderno, México, 2013, p. 43. 
Como se mencionó, este tipo de reformas aisladas no son atribuibles a México solamente. En el contexto latinoamericano, la NGP no ha sido el paradigma dominante en la región. Más bien, las reformas administrativas que incluyen ciertas herramientas de NGP son, por un lado, motivadas por elementos específicos de cada país; en el caso mexicano, la liberalización económica y la pluralidad política influyeron de manera decisiva en las agendas de reformas de la administración pública en los gobiernos panistas. ${ }^{62}$ Por otro lado, los organismos internacionales han jugado también un papel importante en la adopción de herramientas de NGP en la región; por ejemplo, en Colombia se creó el Sistema de Evaluación y Seguimiento para apoyar el manejo de los créditos que otorgó el Banco Mundial y el Banco Interamericano de Desarrollo.

La adopción de estas buenas prácticas o experiencias neogerenciales exitosas busca incidir en temas como la planificación, la presupuestación, la evaluación, el uso de indicadores, la atención al usuario, entre otros. De ahí que algunos de estos temas sigan vigentes en la administración actual, como el de la evaluación o el presupuesto. El caso de la autonomía del Consejo Nacional de Evaluación de la Política de Desarrollo Social es un intento por reformar o continuar con la misma temática de cambio en la administración pública, que no se vincula a un proyecto integral o coherente de reforma administrativa y que no necesariamente parte de las especificidades del caso mexicano.

\section{BibLIOGRAFÍA}

Arellano Gault, David, "Nueva Gestión Pública: ¿el meteorito que mató al dinosaurio? Lecciones para la reforma administrativa en países como México", Revista del Centro Latinoamericano de Administración para el Desarrollo, núm. 23, 2002.

Castro, Manuel Fernando, Gladys López-Acevedo, Gita Beker Busjeet y

${ }^{62}$ Guillermo Cejudo, "Los motores de cambio en la administración pública mexicana”, en María del Carmen Pardo y Ernesto Velasco, La gerencia pública en América del Norte, México, El Colegio de México, 2009, pp. 131-158. 
Ximena Fernández Ordoñez, El Sistema de MẼE en México: un salto del nivel sectorial al nacional, Serie de documentos de trabajo DCE, Banco Mundial, 2009.

Cejudo, Guillermo M., "Explaining Change in the Mexican Public Sector: the Limits of the New Public Management”, International Review of Administrative Sciences, vol. 74, 2008, pp. 111-127.

_ na”, en María del Carmen Pardo y Ernesto Velasco, La Gerencia Pública en América del Norte, México, El Colegio de México, 2009, 131-158.

Dussauge, Mauricio, "La literatura comparada sobre reformas administrativas”, Gestión y política pública, vol. 18, núm. 2, 2008.

-, "Paradoxes of Public Sector Reform: The Mexican Experience (2000-2007)", International Public Management Review, vol. 9, núm. 1, 2008.

Gobierno de la República, Programa para un Gobierno Cercano y Moderno, México, 2013.

Guerrero, Juan Pablo, "La reforma a la administración pública mexicana en el nuevo régimen político. ¿Por dónde empezar? Ideas para la creación de un gobierno transparente, responsable y cercano a la ciudadanía”, México, Centro de Investigación y Docencia Económicas, Cuadernos de Trabajo, 2001.

Hinojosa Cruz, Adriana Verónica, "Configuración histórica de la nueva Ley Federal de Presupuesto y Responsabilidad Hacendaria”, XI Congreso Internacional del CLAD sobre la Reforma del Estado y de la Administración Pública, Ciudad de Guatemala.

Hood, Christopher y Martin Lodge, The Politics of Public Service Bargains, Oxford University Press 2006.

Klinger, Donald, "South of the Border': Progress and Problems in Implementing New Public Management Reforms in Mexico Today", The American Review of Public Administration, núm. 30, 2000.

Mumme, Stephen P. y Debra J. Little, "Leadership, Politics, and Administrative Reform at the United States Section of the International Boundary and Water Commission, United States and Mexico", The Social Science Journal, vol. 47, núm. 2, 2010, pp. 252-270.

Muñoz, Ramón, Innovación gubernamental, México, FCE, 2004 (Sección de Obras de Administración Pública). 
Plan Nacional de Desarrollo 2007-2012, Presidencia de la República, México, 2007, en http://www.pnd.calderon.presidencia.gob.mx

Peters, Guy, The Future of Governing, Lawrence, University of Kansas Press, 2001.

Presidencia de la República, "Decreto que establece las medidas de austeridad y reducción del gasto en la administración pública federal", Diario Oficial de la Federación, 4 de diciembre de 2006, http://www. firco.gob.mx/firco/docs/DecretoDeAusteridad.pdf

Sánchez González, José Juan, "El cambio institucional en la reforma y modernización administrativa de la administración pública mexicana”, Gestión y Política Pública, vol. 18, núm. 1, 2009.

, "La propuesta de modernización administrativa del gobierno de Felipe Calderón”, Revista de Administración Pública, vol. 45, 2010.

Schneider, Ben y Blanca Heredia (coords.), Reinventing Leviathan: The Politics of Administrative Reform in Development Countries, North-South Carolina Press, 2003.

Secretaría de la Función Pública, Programa Especial para la Mejora de la Gestión en la Administración Pública Federal 2008-2012, http:// www.funcionpublica.gob.mx/index.php/pmg.html

Secretaría de Hacienda y Crédito Público, Sistema de Evaluación del Desempeño, México, 2007.

— Presupuesto basado en Resultados (PbR) y Sistema de Evaluación del Desempeño (SED), México, Subsecretaría de Egresos, Unidad de Política y Control Presupuestario, 2008.

_, "Programa Anual de Evaluación", en http://www.shcp.gob.mx

Stark, Andrew, "What is the New Public Management", Journal of Public Administration Research and Theory: J-PART, vol. 11, núm. 1, 2002.

Vega Casillas, Salvador, "El Programa Especial de Mejora de la Gestión", Conferencia Internacional sobre Presupuesto Basado en Resultados, Ciudad de México, 2008.

, "La modernización del gobierno mexicano a partir de la mejora de la gestión: entrevista al Secretario de la Función Pública. Entrevista realizada por José Sosa”, RAP, México, INAP, vol. 43, núm. 1, 2008. 